\title{
Expediting the Diagnosis of Hypertension; The Canadian Hypertension Education Program 2015 Recommendations
}

Norm Campbell MD FRCPC, Mark Gelfer MD, Lyne Cloutier RN PhD, Maxime Lamarre-Cliche MD MSc, Donna McLean RN NP PhD, Raj Padwal MD FRCPC

\begin{abstract}
About the Authors
Norm Campbell is a professor in the Departments of Medicine, Community Health Sciences, and Physiology and Pharmacology and a member of the O'Brien Institute of Public Health and Libin Cardiovascular Institute of Alberta at the University of Calgary in Calgary, Alberta. Mark Gelfer is a clinical assistant professor in the Department of Family Practice at the University of British Columbia, in Vancouver, British Columbia. Lyne Cloutier is a professor in the Department of Nursing, at the Université du Québec à Trois-Rivières. Maxime Lamarre-Cliche is an internist, at the Institut de Recherches Cliniques de Montréal, at the University of Montréal, in Montreal, Quebec. Donna McLean is an adjunct faculty member in the Faculty of Nursing, at the University of Alberta and a sessional faculty member at the Faculty of Health and Community Studies, at MacEwan University in Edmonton, Alberta. Raj Padwal is a professor of medicine and director of the Hypertension Clinic at the University of Alberta, in Edmonton, Alberta. Correspondence can be directed to ncampbel@ucalgary.ca.
\end{abstract}

$\mathrm{T}$ The diagnosis of hypertension is fundamental to the practice of medicine. Increased blood pressure (BP) is the second leading global risk factor for death and disability (behind unhealthy diets), accounting for $18 \%$ of global deaths ( $>9$ million deaths per year). ${ }^{1}$ Contemporary guidelines recommend assessing $\mathrm{BP}$ at every care visit; thus, $\mathrm{BP}$ measurement is probably the most commonly performed diagnostic maneuver in medicine. ${ }^{2}$ Importantly, evolving technology is making the diagnosis of hypertension more accurate, reliable, and rapid.

Although accurate when performed in standardized fashion, auscultatory BP is consistently incorrectly and, hence, inaccurately performed in clinical practice. ${ }^{3}$ Provider education programs have failed to produce consistent and sustained improvements in accuracy. Electronic (oscillometric) BP measurement removes or minimizes many (but certainly not all) measurement errors, such as those due to impaired hearing, missed auscultatory gap, rapid cuff deflation, and terminal digit preference. ${ }^{3}$ Furthermore, oscillometric readings (in studies with home, ambulatory, and automated office readings) are more closely related to vascular risk than are auscultatory readings, when the latter are performed in a routine manner, which they are most of the time. Hence in 2014, the World Hypertension League and, in 2015, the Canadian Hypertension
Education Program (CHEP), endorsed preferential use of electronic (oscillometric) BP assessment. ${ }^{3-4}$ One commonly used means of electronic measurement is a fully automated $\mathrm{BP}$ device (automated office BP or AOBP) that can be deployed to take multiple readings, usually while the patient is alone in an exam room, thereby reducing white coat effect. Readings with AOBP devices of $135 / 85 \mathrm{mmHg}$ and above should be considered high enough to initiate a full assessment to confirm the presence of hypertension, while electronic devices that require manual initiation of each measurement, which is termed office blood pressure measurement (OBPM), or auscultatory blood pressure readings (OBPM) still use a $140 / 90 \mathrm{mmHg}$ threshold (Figure 1). Important to all methods of BP assessment is the need to follow standardized protocols to ensure accuracy.

CHEP has also made a second major change to its diagnostic algorithm to recommend that out-of-office measurement (ambulatory blood pressure measurement [ABPM] or home blood pressure measurement (HBPM), with ABPM preferred) be performed to confirm that an elevated AOBP or OBPM truly represents a diagnosis of hypertension (Figure 1). ${ }^{4}$ Outof-office measurement should be performed early in the evaluation of patients with elevated BP readings (after the first hypertension visit) to expedite the diagnosis of hypertension 


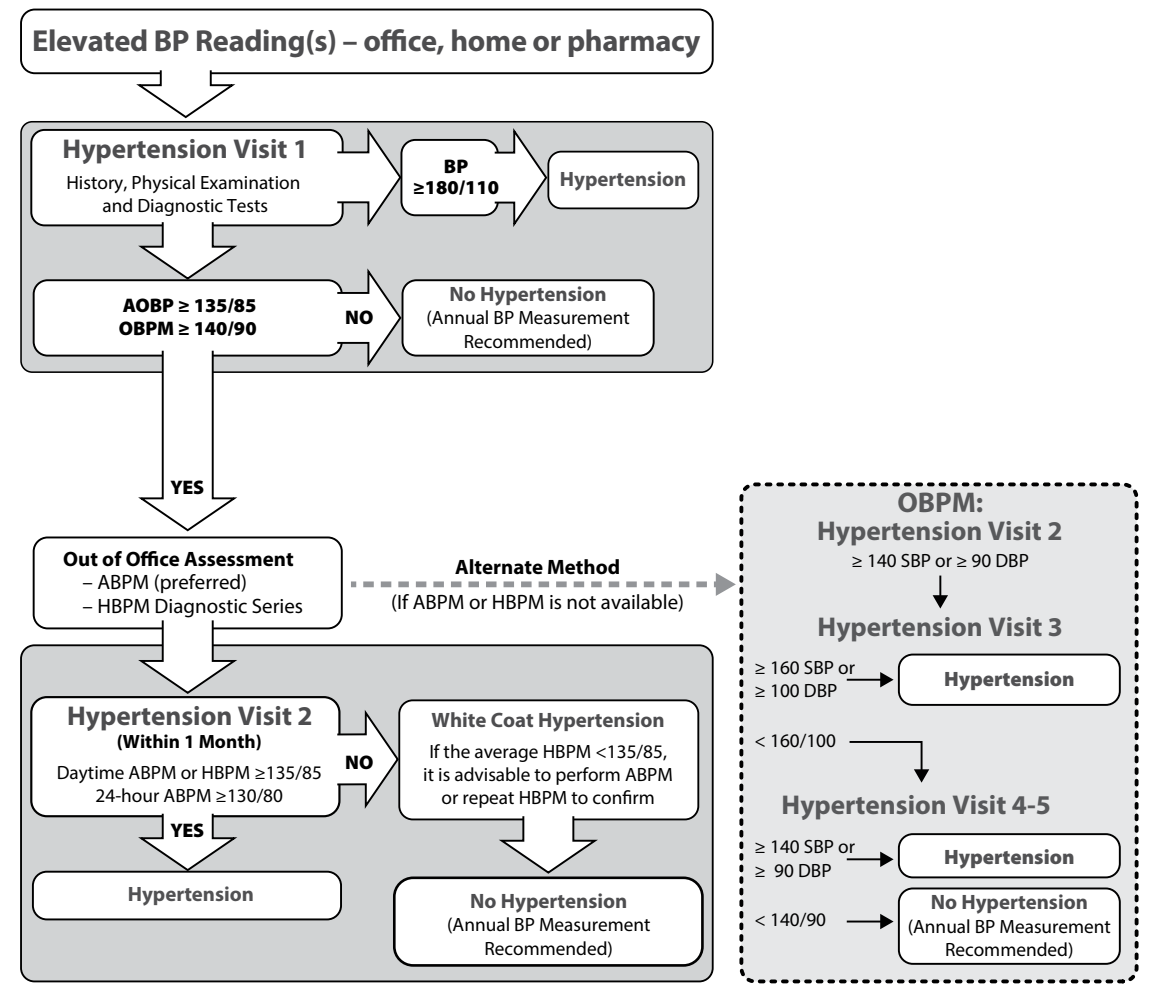

Figure 1. The 2015 Canadian Hypertension Education program diagnostic algorithm for hypertension.

and identify patients with white-coat hypertension. A revised protocol with detailed procedures is also provided in the new CHEP recommendations. As before, people with markedly elevated BP ( $\geq 180 / 110 \mathrm{mmHg})$ or hypertensive emergencies and urgencies can be diagnosed immediately.

After out-of-office measurement is performed, patients identified as having high-normal (130-139/85-89 mmHg) or white coat should be counselled to optimize their health behaviours and followed annually, as they have a high rate of progression to overt hypertension. Patients with white-coat hypertension do not require pharmacologic management for their BP. For clinicians who continue to measure office blood pressure using an auscultatory technique (not recommended), CHEP continues to advise that up to five visits will be required to diagnose hypertension in patients who have initial office readings in stage 1 hypertension range (140-159/90$99 \mathrm{mmHg}$ ).

These new CHEP recommendations are important for clinicians to understand, as they will optimize hypertension care delivery. It is vital that these recommendations be incorporated into undergraduate and post-graduate curricula/OSCE, as well as in continuing medical education programs. Further resources for training and education can be found at www.hypertension.ca. The greatest proportion of undiagnosed hypertension occurs in young Canadians (aged under 40 years), hence screening is important in this subgroup. ${ }^{5}$ Nevertheless, the transition from normal to hypertension occurs at all ages. Canada leads the world in its high rate of hypertension awareness and in treatment and control rates, but still too many are undiagnosed or uncontrolled. ${ }^{5}$

\section{References}

1. Institute for Health Metrics and Evaluation. Global Burdon of Disease Arrow Diagram. Seattle (WA): IHME, 2013. Available at: http://www. healthmetricsandevaluation.org/gbd/visualizations/gbd-arrow-diagram. Accessed Feb 20, 2015.

2. Lindsay P, Conner Gorber S, Joffres M, et al. Recommendations on screening for high blood pressure in Canadian adults. Can Fam Physician 2013;59:92733.

3. Campbell NRC, Berbari AE, Cloutier L, et al. Policy Statement of the World Hypertension League on non-invasive blood pressure measurement devices and blood pressure measurement in the clinical or community setting. J Clin Hypertens 2014;16:320-2.

4. Cloutier L, Daskalopoulou SS, Padwal RS, et al. A new algorithm for the diagnosis of hypertension in Canada. Can J Cardiol 2015;31:549-68.

5. Campbell NRC, McAlister FA, Quan H, Hypertension Outcomes Research Task Force. Monitoring and evaluating efforts to control hypertension in Canada. Why, how and what it tells us about current care gaps. Can J Cardiol 2013;29:564-70. 